\title{
Reusabilidade de objetos educacionais
}

\author{
Liane Margarida Rockenbach Tarouco * \\ Marie-Christine Julie Mascarenhas Fabre \\ Fabrício Raupp Tamusiunas
}

\begin{abstract}
Resumo: A tecnologia de informática e comunicação atualmente permite criar material didático usando multimídia com interatividade que tornam mais efetivos os ambientes de ensino-aprendizagem apoiados nas TICs. Todavia o projeto e desenvolvimento deste tipo de material de apoio demanda muito esforço, mesmo considerando o uso de linguagens de autoração. Isto ensejou o desenvolvimento da estratégia de orientar sua construção na metodologia orientada a objetos. Os recursos educacionais construídos segundo esta estratégia foram denominados objetos educacionais (learning objects) e organismos de padronização como o IEEE (1484.12.1-2002 IEEE Standard for Learning Object Metadata) e ISO (SC 36 WG 2 - Information Technology for Learning, Education and Training) têm grupos trabalhando na elaboração de propostas para sua estruturação e categorização (metadados) de modo a alcançar acessibilidade, interoperabilidade, durabilidade e reusabilidade dos recursos educacionais construídos. Este trabalho pretende apresentar os resultados alcançados numa fase preliminar de criação de um repositório de objetos educacionais reusáveis, a estrutura de metadados criada para sua categorização e o ambiente de implementação do sistema de cadastramento dos objetos educacionais, o qual usa um servidor de diretório baseado no padrão LDAP (Lightweight Directory Access Protocol).
\end{abstract}

Palavras-chave: objetos educacionais, metadados, reusabilidade.

\section{Introdução}

A tecnologia de informática e comunicação atualmente permite criar material didático usando multimídia com interatividade que tornam mais efetivos os ambientes de ensino-aprendizagem apoiados nas TICs. No entanto, o projeto e desenvolvimento desses recursos, mesmo considerando o uso de linguagens de autoração, demandam muito esforço e envolvem grandes investimentos em recursos humanos e financeiros.

Isto ensejou o desenvolvimento da estratégia de orientar sua construção na metodologia orientada a objetos. Os recursos educacionais construídos segundo esta

\footnotetext{
"Professora do Programa de Pós-Graduação em Informática na Educação (PGIE/UFRGS) e da Faculdade de Educação (UFRGS), Diretora do Centro Interdisciplinar de Novas Tecnologias na Educação (CINTED/UFRGS), liane@penta.ufrgs.br.

Pesquisadora do Programa de Pós-Graduação em Informática na Educação (PGIE/UFRGS), Especialista em Informática na Educação (PGIE/UFRGS), marie@pgie.ufrgs.br.

Analista de Sistemas POP-RS/CPD (UFRGS), Bacharel em Ciência da Computação (ULBRA), fabricio@penta.ufrgs.br.
} 
estratégia foram denominados objetos educacionais (learning objects) e organismos de padronização como o IEEE (1484.12.1-2002 Standard for Learning Object Metadata) e ISO (SC 36 WG 2 - Information Technology for Learning, Education and Training) têm grupos trabalhando na elaboração de propostas para sua estruturação e categorização (metadados).

O metadado de um objeto educacional descreve características relevantes que são utilizadas para sua catalogação em repositórios de objetos educacionais reusáveis, podendo ser recuperados posteriormente através de sistemas de busca ou utilizados através de learning management systems (LMS) para compor unidades de aprendizagem.

Além da reusabilidade desses recursos, que possibilita incorporá-los em múltiplas aplicações, destacam-se também outros benefícios da catalogação de objetos educacionais:

* acessibilidade: pela possibilidade de acessar recursos educacionais em um local remoto e usá-los em muitos outros locais;

* interoperabilidade: podendo utilizar componentes desenvolvidos em um local, com algum conjunto de ferramentas ou plataformas, em outros locais com outras ferramentas e plataformas;

* durabilidade: para continuar usando recursos educacionais quando a base tecnológica muda, sem reprojeto ou recodificação.

\section{Objetos educacionais}

Objetos educacionais podem ser definidos como qualquer recurso, suplementar ao processo de aprendizagem, que pode ser reusado para apoiar a aprendizagem. $\mathrm{O}$ termo objeto educacional (learning object) geralmente aplica-se a materiais educacionais projetados e construídos em pequenos conjuntos com vistas a maximizar as situações de aprendizagem onde o recurso pode ser utilizado.

A idéia básica é a de que os objetos sejam como blocos com os quais será construído o contexto de aprendizagem. O projeto e criação destes objetos são realizados usando-se linguagens e ferramentas de autoria que permitem maior produtividade uma vez que a construção dos mesmos demanda elevada quantidade de tempo e recursos, especialmente quando envolvem multimídia.

Objetos educacionais são mais eficientemente aproveitados quando organizados em uma classificação de metadados e armazenados em um repositório integrável a um sistema de gerenciamento de aprendizagem (Learning Management System). A adoção de padrões abertos para este fim é desejável, uma vez que o rápido avanço da tecnologia leva à possível substituição de plataformas de gerenciamento de aprendizagem com maior rapidez do que a desatualização e/ou obsolescência de um objeto educacional, que pode ser atualizado e continuar a ser reusado em outro contexto. A estratégia de adotar padrões abertos também tem como objetivo alcançar independência de plataforma onde os objetos vão ser exibidos/executados permitindo o uso de diferentes sistemas operacionais e plataformas de hardware. Considerando-se as cada vez mais variadas opções para o eLearning, isto é especialmente relevante, pois o ambiente a apoiar a aprendizagem está se expandindo para além do escopo dos computadores atualmente sendo usados, passando a utilizar também computadores de mão portáteis (agendas eletrônicas) e mesmo telefones celulares das novas gerações. 
O compartilhamento de objetos educacionais pode ser condicionado a aspectos legais, financeiros e técnicos e, por este motivo, o acesso aos mesmos pode e deve ser controlado, e este controle de acesso também deve utilizar soluções baseadas em padrões abertos. A solução advogada pela equipe é a de usar um serviço de diretórios padronizado para catalogar os objetos educacionais. Este serviço será descrito na próxima sessão bem como o projeto em desenvolvimento.

O repositório de objetos educacionais pode ser local (uma só instituição) ou distribuído (consórcio de instituições). Existem atualmente diversos consórcios de instituições acadêmicas organizando repositórios de objetos educacionais. Um dos esforços mais relevantes é o da Advanced Distributed Learning (ADL) Initiative, patrocinada pelo Office of the Secretary of Defense (OSD), que é um esforço conjunto do governo norte-americano, indústria e academia para estabelecer um novo contexto educacional que permita a interoperabilidade de ferramentas de aprendizagem e conteúdos em escala global (ADL, 2003).

A descrição dos atributos de catalogação dos objetos (metadados) deve permitir sua pesquisa e recuperação por diferentes critérios. Ferramentas de suporte à aprendizagem podem interagir diretamente com o repositório, selecionando e recuperando objetos, combinando-os de modo a compor unidades de suporte a aprendizagem que atendam a necessidades individuais de cada estudante.

O tamanho/granulidade dos objetos educacionais deve ser escolhido de modo a maximizar seu reuso. Embora objetos educacionais maiores sejam mais fáceis de administrar são menos fáceis de recontextualizar para outros cenários de aprendizagem diferentes daqueles para os quais foram inicialmente previstos. Objetos educacionais menores podem ser mais precisamente definidos, são mais fáceis de recontextualizar mas demandam esforço para organizar com vistas a facilitar sua localização.

\section{Servidor de diretórios para registrar objetos educacionais}

Com o aumento no tamanho das redes e as constantes mudanças pelas quais as redes passam, os usuários passam a necessitar um serviço que permita um acesso transparente ao usuário aos recursos da rede. Um Serviço de Diretórios é responsável por permitir que o usuário possa consultar ou navegar em diretórios de usuário, organizações ou recursos, sem ter a necessidade de conhecer detalhes sobre os objetos armazenados nestes diretórios. Indivíduos e organizações podem usar um serviço de diretórios para tornar disponível um amplo conjunto de informações sobre eles próprios e sobre os recursos que desejam oferecer para uso na rede. Usuários podem pesquisar o diretório em busca de informações específicas, tendo somente conhecimento parcial sobre o seu nome, estrutura ou conteúdo.

A ITU (International Telecommunicatins Unit) e a ISO (International Standards Organization) definiram um conjunto de padrões para um Serviço de Diretório de rede, chamado de X.500 (ITU, 1988 e ISO, 1988). O padrão X.500 especifica um sistema de Diretório distribuído que atende a consultas sobre objetos da rede. Este sistema engloba uma base de dados constituída de nomes e, para cada nome, um conjunto de propriedades a ele associadas. O padrão define o serviço como de acesso a informações sobre entidades do mundo real, porém ele também pode ser utilizado para acessar informações sobre serviços de hardware e software. O serviço de Diretório pode ser também usado para apoiar a definição de grupos de objetos, para autenticação de 
usuários e para funções de gerenciamento de redes. O padrão X.500 define o Diretório como uma coleção de sistemas abertos que cooperam para manter uma base de dados lógica com informações sobre um conjunto de objetos do mundo real. Os usuários do Diretório, incluindo pessoas e programas, podem ler e modificar as informações, ou parte dela, se tiverem permissão para isto (Weider e Reynolds, 1992).

A recomendação X.500 é definida para suportar um diretório amplamente distribuído. Uma maneira de alcançar a distribuição é definir uma rede de processos clientes e servidores. Cada processo pode residir em um computador diferente e os processos se comunicam uns com os outros através de protocolos bem definidos. Processos clientes requisitam serviços dos servidores, que fornecem estes serviços.

No modelo funcional são definidos dois tipos de processos ou agentes:

* Agentes do Usuário de Diretórios (AUD)

* Agentes do Serviço de Diretórios (ASD)

Um Agente do Usuário de Diretórios atua em benefício de um único usuário de Diretório. Quando uma pessoa ou processo deseja utilizar o Diretório interage com seu AUD. O AUD então desencadeia as ações apropriadas. O Agente de Usuário do Diretório está concentrado no primeiro nível de abstração e trata da maneira pela qual o usuário interage com o Diretório. A natureza desta interação entre os dois componentes foi padronizada, e envolve um conjunto de operações que pode ser executada no Diretório por um usuário do Diretório. Este protocolo é denominado Protocolo de Acesso ao Diretório (DAP).

Um Agente do Sistema de Diretório é um processo de aplicação OSI que é parte do Diretório é cujo papel é fornecer acesso à base de informações para Agentes de Usuário do Diretório e/ou para outros ASDs. Um ASD pode usar informações armazenadas em seu banco de dados local ou pode interagir com outros ASDs para responder a requisições.

Todos os serviços de Diretório são providos ao usuário pelo Diretório em resposta a pedidos dos AUDs. Existem pedidos que permitem conexão ao diretório, interrogação do Diretório e serviços para modificação do Diretório. Cada pedido pode estar acompanhado por informações para suportar mecanismos de segurança para proteger as informações do Diretório. Estas informações podem incluir pedidos de usuário para vários tipos de proteção, como por exemplo, assinatura digital do pedido, junto com informação para auxiliar um interlocutor correto a verificar a assinatura.

Existem dois protocolos usados pelo Diretório. O Protocolo de Acesso ao Diretório (PAD) é usado em interações entre um AUD e um ASD. Já o Protocolo do Sistema de Diretórios (PSD) é usado em interações entre dois ASD.

O interesse da Internet no serviço definido pela recomendação X.500 tinha como obstáculo a complexidade dos protocolos especificados na recomendação X.500 para a interoperação dos servidores de diretórios, pois foi previsto que toda a pilha OSI (Open System Interconnection) fosse usada. Um esforço de simplificação foi feito sobre o protocolo DAP resultando no que foi denominado LDAP (Lighweight Directory Access Protocol) um protocolo de acesso ao servidor de diretório mais leve, menos complexo, com as mensagens do protocolo de aplicação transportadas diretamente pela camada TCP (Transport Contro Protocol) da arquietura da Internet (Hodges e Morgan, 2002; Stokes et al. 2002). 


\section{O projeto CESTA}

O projeto CESTA - Coletânea de Entidades de Suporte ao uso de Tecnologia na Aprendizagem foi iniciado com vistas a sistematizar e organizar o registro dos objetos educacionais que vinham sendo desenvolvidos pela equipe para cursos de capacitação em Gerência de Redes, Videoconferência e no Pós-Graduação Lato-sensu Informática na Educação. Todos estes cursos têm sido desenvolvidos em modalidade a distância e material didático de apoio foi projetado e construído para apoiar as atividades de aprendizagem. A busca e recuperação dos recursos de aprendizagem é também um aspecto importante neste contexto. Os materiais desenvolvidos, principalmente os que usam multimídia educacional, precisam ser organizados e armazenados com vistas a seu acesso on-line e adequadamente catalogados para que possam ser recuperados quando e como necessário. Visando a possibilidade de reutilização de tais recursos foi projetado e implementado um serviço de diretórios para permitir o registro de objetos educacionais.

Uma especificação inicial de metadados para os objetos educacionais foi elaborada e, a partir da mesma, um serviço de registro e catalogação de objetos educacionais foi colocado em operação, usando um servidor LDAP. 


\section{Exemplos de recursos educacionais produzidos são:}

* vídeos sincronizados com material de apresentação;

* vídeos com demonstrações e/ou simulações;

* CBT - Computer Based Training (ToolBook);

* WBT - Web Based Training (Director, Flash, etc.);

* material interativo construído com programas de apoio para auto-avaliação usando Java e outros mecanismos disponíveis nos ambientes de autoria de courseware, tais como Java Builder.

Os materiais (multimídia educacional) desenvolvidos estão sendo organizados e armazenados em servidores WWW e servidores de vídeo sob demanda. Estes recursos estão sendo catalogados para fins de disponibilização e reuso. A catalogação está sendo feita em consonância com normas de padronização internacionais (IEEE P1484 Leaning Objects Metadata) e os dados de catalogação serão disponibilizados em diretório on-line (servidor LDAP do diretório do projeto DUNGA).

A partir da análise das especificações de metadados para os objetos educacionais elaboradas pelo Learning Technology Standards Committee do IEEE, foi construída uma especificação com atributos para descrever os objetos educacionais, baseado no padrão de metadados para learning objects LTSC/IEEE. A especificação de metadados criada para a categorização dos objetos educacionais no repositório foi elaborada em consonância com a norma IEEE 1484.12.1 (Learning Objects Metadata). A norma especifica atributos agrupados em 9 (nove) categorias para descrição de um objeto educacional. A utilização dos atributos é opcional, ou seja, uma estrutura de metadados, em conformidade com a norma, pode não conter todos os atributos (IEEE/LTSC, 2002).

Para a implementação do sistema de cadastramento dos objetos educacionais, foram utilizadas 5 (cinco) categorias na especificação dos metadados: geral, ciclo de vida, técnica, educacional e direitos.

A categoria geral agrupa informações gerais que descrevem o objeto. Os seguintes atributos foram utilizados:

* Identificador - identificação única do objeto;

* Título - nome dado ao objeto;

* Idioma - idioma utilizado no objeto (português, inglês, espanhol, francês);

* Descrição - descrição textual do conteúdo do objeto;

* Palavras-chave - palavra(s)-chave descrevendo os tópicos do objeto.

A categoria ciclo de vida agrupa informações que descrevem as características relacionadas ao histórico e estado atual dos objetos e todos aqueles que o têm afetado durante sua evolução. Os seguintes atributos foram utilizados:

* Versão - a versão/edição do objeto;

* Status - estado atual do objeto (rascunho, revisado, editado, indisponível);

* Tipo de contribuição - autor, editor, desconhecido, iniciador, designer gráfico, técnico, provedor de conteúdo, roteirista, designer instrucional, especialista em conteúdo;

* Entidades que contribuiram - pessoas e/ou organizações que contribuiram na evolução do objeto; 
* Data - data da contribuição.

A categoria técnica agrupa os requisitos e características técnicas do objeto. Os seguintes atributos foram utilizados:

* Formato - formato de todos os componentes do objeto (MIME types), este atributo pode ser usado para identificar o programa necessário para acessar o objeto;

* Tamanho - tamanho do objeto em bytes;

* Localização - URL (Universal Resource Locator) do objeto;

* Tipo de tecnologia - sistema operacional e navegador (relacionado com o atributo nome da tecnologia);

* Nome da tecnologia - PC-DOS, MS-Windows, Mac-OS, Multi-OS, Unix ou Nenhum para sistema operacional e Netscape, MS-Internet Explorer, Opera, Amaya ou Nenhum para navegador;

* Duração - tempo de duração (utilizado para sons, vídeos, animações).

A categoria educacional agrupa as características educacionais e pedagógicas do objeto. Os seguintes atributos foram utilizados:

* Tipo de interatividade - modo predominante de aprendizagem (ativa, expositiva, mista);

* Recurso de aprendizagem: tipo específico do objeto (exercício, simulação, questionário, diagrama, figura, gráfico, índice, slide, tabela, teste, experiência, texto, problema, auto-avaliação, palestra);

* Nível de interatividade - grau de interatividade (muito baixo, baixo, médio, alto, muito alto);

* Usuário final esperado - tipo de usuário para o qual foi desenvolvido o objeto (professor, autor, aluno, gerenciador);

* Ambiente de utilização - escola, faculdade, treinamento, outro;

* Faixa etária - idade do usuário final esperado;

* Descrição - comentários sobre como esse objeto deve ser usado.

A categoria direitos agrupa os direitos de propriedade intelectual e as condições de uso do objeto. Os seguintes atributos foram utilizados:

*Custo - se a utilização do objeto requer pagamento (sim ou não);

*Direito autoral - se há restrições de direito autoral para o uso do objeto (sim ou não);

* Condições de uso - comentários sobre as condições de uso do objeto.

Utilizando a especificação de metadados proposta, foi implementado o sistema para cadastro e consulta de objetos educacionais conforme apresentado na próxima seção.

\section{A implementação do projeto}

Para a construção deste sistema, utilizou-se, como recurso de armazenamento e consulta, a estrutura de diretórios LDAP (Lightweight Directory Access Protocol). LDAP é um protocolo aberto para acessar serviços de diretórios X.500.

O LDAP é a alternativa leve para o Protocolo de Acesso a Diretórios X.500 (X.500 Directory Access Protocol - DAP) para uso na Internet. Ele usa a pilha TCP/IP ao invés da complexa pilha OSI. Ele também possui outras simplificações, como a representação da maioria dos valores dos atributos como texto e muitos itens do 
protocolo como strings textuais, que são designadas a facilitar a implementação de clientes.

Para a criação da base LDAP foi necessária a especificação dos atributos descritos na norma IEEE 1484.12.1 na linguagem ASN.1 (Abstract Syntax Notation One) e sua posterior incorporação ao objeto lom (Learning Object Model), criado e usado para o cadastro dos objetos educacionais. Para identificar os atributos e objetos foi usado o OID (Object Identificator) 1.2.6.1.9.100, designando pelo IANA (Internet Assigned Numbers Authority) para a UFRGS (Universidade Federal do Rio Grande do Sul).

Os primeiros cinco dígitos do OID acima citado são da UFRGS e o número 100 que vez após este identificador é o número usado para designar o objeto lom.

A tabela 1 mostra um exemplo de um atributo utilizado pelo objeto lom.

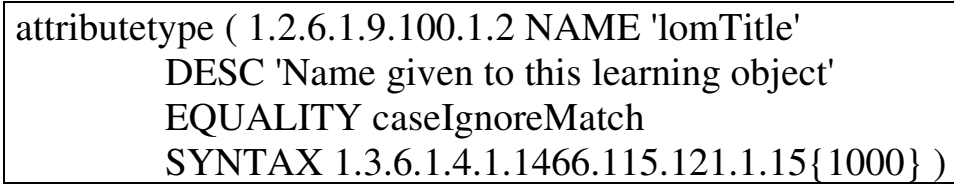

\section{Tabela 1 - Exemplo de atributo}

O identificador do atributo lomTitle, mostrado na tabela 1, possui o identificador 1.2.6.1.9.100.1.2. O número que vem após o OID do objeto é indicativo do código do atributo na norma IEEE 1484.12.1. O campo DESC é uma descrição do atributo. O campo SYNTAX, que possui o identificador 1.3.6.1.4.1.1466.115.121.1.15 (indica que o atributo é uma string), diz que o limite é de 1000 caracteres.

Para cada um dos atributos descritos na norma do IEEE, foi criada uma entrada de atributo dentro do servidor LDAP. A tabela 2 mostra o nome todos os atributos criados e importados pelo objeto lom.

\begin{tabular}{|l} 
objectclass ( 1.2.6.1.9.100.0 NAME 'lom' SUP top STRUCTURAL \\
MUST ( lomIdentifier ) \\
MAY ( lomGeneral \$ lomTitle \$ lomLanguage \$ \\
lomDescription \$ lomKeyword \$ lomLifeCycle \$ lomVersion \$ \\
lomStatus \$ lomContribute \$ lomRole \$ lomEntity \$ lomDate \$ \\
lomTechnical \$ lomFormat \$ lomSize \$ lomLocation \$ \\
lomRequirement \$ lomOrComposite \$ lomType \$ lomDuration \$ \\
lomEducational \$ lomName \$ lomInteractivityType \$ \\
lomLearningResourceType \$ lomInteractivityLevel \$ \\
lomSemanticDensity \$ lomIntendedEndUserRole \$ lomContext \$ \\
lomTypicalAgeRange \$ lomEducationalDescription \$ lomRights \$ \\
lomCost \$ lomCopyrightAndOtherRestrictions \$ lomRightsDescription \\
) )
\end{tabular}

Tabela 2 - Descrição do objeto lom

O exemplo da tabela 2 mostra que o objeto necessita obrigatoriamente do atributo predefinido lomIdentifier e todos os demais que estão no campo MAY.

Para a implantação do servidor LDAP, foi utilizado o software OpenLDAP (OpenLDAP, 2003). Para a criação da interface de consulta foram utilizadas as 
linguagens PHP (PHP, 2003) como SSI (Server Site Included), e DHTML e JavaScript como CSL (Client Site Included).

Para a parte de implementação, o PHP foi usado para receber as informações vindas do formulário de cadastro e fazer a sua inclusão dentro do sistema LDAP. Este mesmo recurso foi utilizado para a parte de consulta, recebendo as informações vindas do formulário de consulta e fazendo uma busca da base de dados LDAP.

O sistema operacional utilizado para o protótipo foi o RedHat Linux versão 7.3. O servidor web, para o suporte as aplicações SSI, utilizado foi o Apache versão 1.3.27. A versão de PHP utilizada foi a 4.3.1.

As entradas de dados no sistema LDAP são realizadas através de uma interface WEB. A figura 1 mostra esta interface.

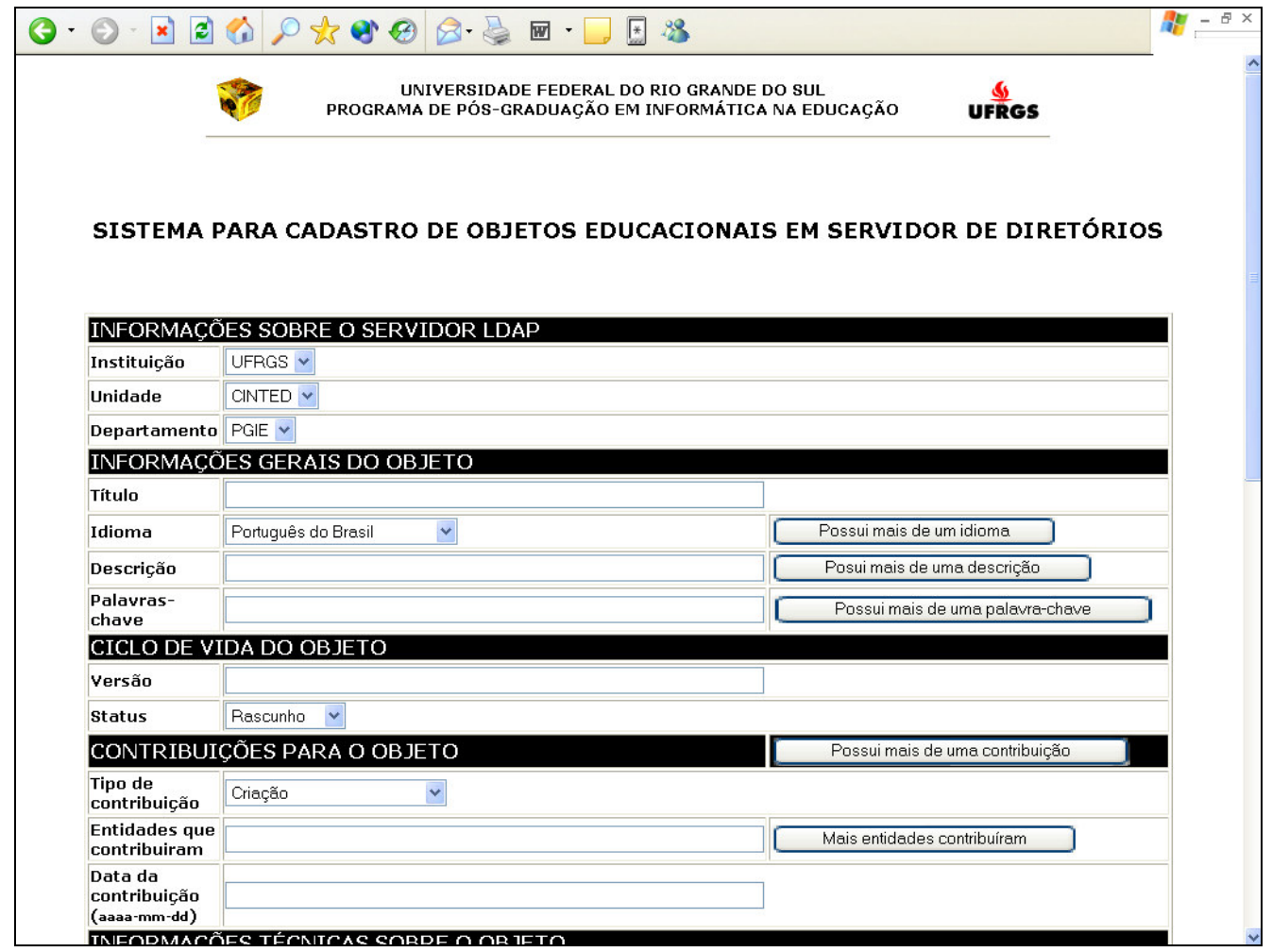

Figura 1 -Tela de cadastro

Assim como o cadastro, a consulta também é realizada utilizando uma interface WEB. A figura 2 mostra o retorno de uma consulta. 


\begin{tabular}{|c|c|c|}
\hline \multicolumn{3}{|c|}{ 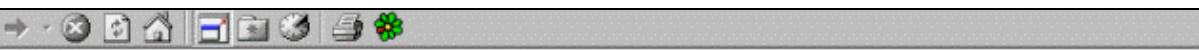 } \\
\hline 9 & $\begin{array}{l}\text { UNIVERSIDADE FEDERAL DO RIO GRANDE DO SUL } \\
\text { PROGRAMA DE PÓS-GRADUAÇÁO EM INFORMÁTICA NA EDUCAÇOA }\end{array}$ & UFRGS \\
\hline \multicolumn{3}{|c|}{ Resultado da Pesquisa } \\
\hline \multicolumn{3}{|c|}{ Informaçôes sobre o objeto lom000001 } \\
\hline Titulo & Etiquetas para videoconferências & \\
\hline Idioma & EN-US & \\
\hline Idioma & PT-BR & \\
\hline \multicolumn{3}{|c|}{ CICLO DE VIDA DO OBJETO } \\
\hline Versằ & 1.0 & \\
\hline Status da versäo 1.0 & final & \\
\hline \multicolumn{3}{|c|}{ CONTRUBUIÇŌES PARA O OBJETO } \\
\hline Entidade que contribuiu & Universidade de Washington & \\
\hline Tipo de contribuifäo & creator & \\
\hline Data & $2001-01-01$ & \\
\hline Entidade que contribuiu & Universidade Federal do Rio Grande do Sul & \\
\hline 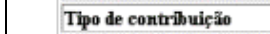 & editor & \\
\hline Data & $2002-06-01$ & \\
\hline \multicolumn{3}{|c|}{ INFORMAÇOZES TÉCNICAS SOBRE O OBJETO } \\
\hline Fonnato & $\mid$\begin{tabular}{l|l} 
audio/x-pn-realaudio \\
\end{tabular} & \\
\hline Tamanho (em bytes) & 2000 & \\
\hline Localizaçäo & http://wwnx pgie ufrgs br/videoconferencia/videoconference m & \\
\hline \multicolumn{3}{|c|}{ REQUISITOS TÉCNICOS PARA FUNCIONALIDADE DO OBJETO } \\
\hline Tipo da Tecnologia & \begin{tabular}{|l|l} 
navegator & \\
\end{tabular} & \\
\hline Nome da Tecnologia & netscape navegator & \\
\hline
\end{tabular}

Figura 2 - Tela de consulta

\section{Conclusões}

A iniciativa ora descrita, de criar um repositório de objetos educacionais para fins de reuso não representa uma iniciativa isolada, mas está em consonância com tendências internacionais. A construção de repositórios interoperáveis de objetos educacionais permitirá o desenvolvimento de sistemas de aprendizagem adaptativos capazes de montar conteúdos sob demanda para prover aos estudantes situações de aprendizagem e apoio em qualquer momento e a partir de qualquer lugar.

Todos estes avanços nos elementos que são mediadores na comunicação atuam como catalisadores de mudanças no cenário educacional. Da aprendizagem apoiada em texto linear, seqüencial, dependente do professor, os personagens atuantes neste novo contexto passam para um processo de aprendizagem por demanda num contexto de multimídia e hipertexto onde a navegação é decidida dinamicamente pelo estudante e que, principalmente, não fica mais restrita ao que o lhe é apresentado pelo professor ou pela escola. Ao contrário, esta serve apenas de base para um processo de crescimento no qual o estudante tem a oportunidade de tomar as rédeas do controle de sua aprendizagem e sair em busca das condições que o ajudarão a crescer, construindo seu conhecimento através de processos de assimilação/acomodação segundo proposto por Piaget (1976). 


\section{Referências bibliográficas}

Advanced Distributed Learning (ADL). Disponível em: http://www.adlnet.org. Acesso em: 11 fev. 2003.

HODGES, J. e MORGAN, R. RFC 3377 Lightweight Directory Access Protocol (v3): Technical Specification.. September, 2002.

IEEE Learning Technology Standards Committee (LTSC). Draft Standard for Learning Object Metadata (IEEE 1484.12.1-2002). Julho de 2002. Disponível em: http://ltsc.ieee.org/doc/wg12/LOM_1484_12_1_v1_Final_Draft.pdf. Acesso em: 02 fev. 2003.

International Telecommunicatins Unit. The Directory: Overview of Concepts, Models and Service. ITU Recommendation X.500, 1988.

International Standards Organization. ISO Information Processing Systems -- Open Systems Interconnection -- The Directory: Overview of Concepts, Models and Service. ISO/IEC JTC 1/SC21; International Standard 9594-1, 1988.

OpenLDAP Software. Disponível em: http://www.openldap.org. Acesso em: 12 jan. 2003.

PHP: Hypertext Preprocessor. Disponível em http://www.php.net. Acesso em: 17 fev. 2003.

PIAGET, J; INHELDER, B. Da lógica da criança à lógica do adolescente. São Paulo: Pioneira, 1976.

STOKES, E.; WEISER, R.; MOATS, R.; HUBER, R. RFC 3384 Lightweight Directory Access Protocol (version 3) Replication Requirements. October, 2002.

WEIDER, C.; REYNOLDS, J. RFC1308 Executive Introduction to Directory Services Using the X.500 Protocol. Março, 1992. 